\title{
Erratum to: Quantitatively evaluating detoxification of the hepatotoxic microcystin-LR through the glutathione (GSH) pathway in SD rats
}

\author{
Xiaochun Guo ${ }^{1,2,3} \cdot$ Liang Chen ${ }^{1,4} \cdot$ Jun Chen ${ }^{1} \cdot$ Ping Xie $^{1,3} \cdot$ Shangchun $\mathrm{Li}^{1,4}$. \\ Jun $\mathrm{He}^{1} \cdot$ Wei $\mathrm{Li}^{1,4} \cdot$ Huihui Fan ${ }^{1,3} \cdot$ Dezhao Yu ${ }^{1,4}$ Cheng Zeng ${ }^{1,3}$
}

Published online: 23 January 2016

(C) Springer-Verlag Berlin Heidelberg 2016

Erratum to: Environ Sci Pollut Res (2015) 22:19273-19284

DOI 10.1007/s11356-015-5531-2

The original version of this article unfortunately contains mistakes.

Jun Chen is the sole corresponding author

Xiaochun Guo and Liang Chen contributed equally to the original article.

$\triangle$ Jun Chen

chenjun@ihb.ac.cn

1 Donghu Experimental Station of Lake Ecosystems, State Key Laboratory of Freshwater Ecology and Biotechnology, Institute of Hydrobiology, Chinese Academy of Sciences, Wuhan 430072, Hubei, China

2 Research Center of Lake Environment, Chinese Research Academy of Environmental Sciences, Beijing 100012, China

3 College of Fisheries, Huazhong Agricultural University, Wuhan 430070, Hubei, China

4 University of Chinese Academy of Sciences, Beijing 100049, China 